\title{
Three-body breakup in dissociative electron attachment to the water molecule
}

\author{
Daniel J. Haxton, ${ }^{1, *}$ Thomas N. Rescigno, ${ }^{2, \dagger}$ and C. William McCurdy ${ }^{2,3, \ddagger}$ \\ ${ }^{1}$ JILA, the University of Colorado, Boulder and NIST, Boulder CO 80305 \\ ${ }^{2}$ Lawrence Berkeley National Laboratory, Chemical Sciences, Berkeley, California 94720 \\ ${ }^{3}$ Departments of Applied Science and Chemistry, \\ University of California, Davis, California 95616
}

\begin{abstract}
We report the results of $a b$ initio calculations on dissociative electron attachment (DEA) to water that demonstrate the importance of including three-body breakup in the dissociation dynamics. While three-body breakup is ubiquitous in the analogous process of dissociative recombination, its importance in low-energy dissociative electron attachment to a polyatomic target has not previously been quantified. Our calculations, along with our earlier studies of DEA into two-body channels, indicate that three-body breakup is a major component of the observed $\mathrm{O}^{-}$cross section. The local complex potential model provides a generally accurate picture of the experimentally observed features in this system, reproducing some quantitatively, others qualitatively, and one not at all.
\end{abstract}

PACS numbers: 03.65.Nk, 34.80.-i, 34.80.Ht

Dissociative electron attachment (DEA) to the water molecule,

$$
\mathrm{H}_{2} \mathrm{O}+e^{-} \rightarrow \begin{cases}\mathrm{H}+\mathrm{OH}^{-} & 3.27 \mathrm{eV} \\ \mathrm{H}_{2}+\mathrm{O}^{-} & 3.56 \mathrm{eV} \\ \mathrm{H}^{-}+\mathrm{OH} \quad\left(X^{2} \Pi\right) & 4.35 \mathrm{eV} \\ \mathrm{H}+\mathrm{H}+\mathrm{O}^{-} & 8.04 \mathrm{eV} \\ \mathrm{H}^{-}+\mathrm{OH}^{*} \quad\left({ }^{2} \Sigma\right) & 8.38 \mathrm{eV} \\ \mathrm{H}^{-}+\mathrm{H}+\mathrm{O} & 8.75 \mathrm{eV}\end{cases}
$$

is a resonant process that involves the capture of a free electron into a transient negative ion state which subsequently dissociates to produce neutral and ionic fragments. Previous experimental [1-10] and theoretical studies [11-19] have characterized the various breakup channels and the three metastable anion states involved in the DEA process - the ${ }^{2} B_{1},{ }^{2} A_{1}$, and ${ }^{2} B_{2}$ electronic Feshbach resonances with peaks near 6.4, 8.4 and $12 \mathrm{eV}$, respectively. DEA in water is governed by complex electronic and nuclear dynamics involving, as we have previously shown [16], anion surfaces that interact via conical intersection and Renner-Teller effects. Our previous studies on this system [14-19], involving anion surfaces computed $a b$ initio and nuclear dynamics calculations carried out in full dimensionality within the local complex potential model, succeded in giving a quantitatively accurate description of the major DEA channel - $\mathrm{H}^{-}+$ $\mathrm{OH}$ production through the ${ }^{2} B_{1}$ state - as well as a qualitatively accurate description of many features associated with the minor channels, with two notable exceptions. Our calculations significantly underestimated the cross section for $\mathrm{O}^{-}$production via the ${ }^{2} B_{2}$ state and produced a zero result for $\mathrm{O}^{-}$production via the ${ }^{2} A_{1}$ state,

\footnotetext{
*Electronic address: dhaxton@jila.colodado.edu

$\dagger$ Electronic address: tnrescigno@lbl.gov

${ }^{\ddagger}$ Electronic address: cwmccurdy@lbl.gov
}

although the latter process has been clearly observed experimentally. The purpose of this communication is to resolve these two discrepancies between theory and experiment.

Our work to date has only considered the breakup of transient $\mathrm{H}_{2} \mathrm{O}^{-}$species into diatomic fragments, $\mathrm{H}^{-}+$ $\mathrm{OH}$ and $\mathrm{O}^{-}+\mathrm{H}_{2}$, and did not treat three-body breakup. While three-body breakup is known to be important in dissociative recombination of polyatomic ions, where three-body channels are generally open even at threshold, for DEA the process has yet to be quantified. The threebody channels $\mathrm{H}^{-}+\mathrm{H}+\mathrm{O}$ and $\mathrm{O}^{-}+\mathrm{H}+\mathrm{H}$ open up within the second $\left({ }^{2} \mathrm{~A}_{1}\right)$ DEA peak, but are energetically closed for the lowest ${ }^{2} \mathrm{~B}_{1}$ resonance. In an experiment on DEA to $\mathrm{D}_{2} \mathrm{O}$, Curtis and Walker [9] observed the opening of the lower three-body channel, as discerned through the kinetic energy distribution of the $\mathrm{O}^{-}$fragment. However, because of the large $\mathrm{O}$ atom $/ \mathrm{H}_{2}$ mass ratio, such a measurement is difficult and a quantitative experimental determination of the final-state branching ratios among the two- and three-body breakup channels has yet to be published.

There is strong reason to believe that three-body breakup is important for describing DEA leading to $\mathrm{O}^{-}$ production. In particular, examination of the shape of the ${ }^{2} \mathrm{~A}_{1}$ surface suggests that three-body breakup may be the key to describing $\mathrm{O}^{-}$production via this resonance. The ${ }^{2} \mathrm{~A}_{1}\left(1^{2} \mathrm{~A}^{\prime}\right)$ electronic surrface slopes downward toward linear $\mathrm{H}-\mathrm{O}-\mathrm{H}$ geometry and is dissociative along the $\mathrm{OH}$ bonds. It provides a path for symmetric dissociation of the $\mathrm{OH}$ bonds to produce $\mathrm{O}^{-}+\mathrm{H}+\mathrm{H}$, but also slopes downward toward the $\mathrm{H}^{-}+\mathrm{OH}$ asymptotes. Similarly, the ${ }^{2} \mathrm{~B}_{2}$ diabatic surface, which equals the ${ }^{2} \mathrm{~B}_{2}$ adiabatic surface for $\mathrm{C}_{2 v}$ geometries, provides a path toward symmetric dissociation through the conical intersection with the ${ }^{2} \mathrm{~A}_{1}$ state. Symmetric dissociation of the ${ }^{2} \mathrm{~B}_{2}$ state from the equilibrium geometry of the neutral leads to the $\mathrm{O}^{-}+\mathrm{H}+\mathrm{H}$ three-body asymptote.

Our methodology is very similar to that used in our previous calculations, so we provide only a brief summary 
here. We use the local complex potential or "boomerang" model[20-24] in which the nuclear motion is determined by a driven Schrodinger equation that describes the dynamics of the metastable $\mathrm{H}_{2} \mathrm{O}^{-}$electronic state(s) that correlate(s) with the neutral + anion fragments:

$$
(E-H) \xi_{\nu_{i}}(\vec{q})=\phi_{\nu_{i}}(\vec{q}, 0),
$$

where the Born-Oppenheimer anion Hamiltonian is

$$
H=K_{\vec{q}}+E_{R}(\vec{q})-\frac{i \Gamma(\vec{q})}{2}
$$

the nuclear degrees of freedom are collectively denoted by $\vec{q}$, and the nuclear kinetic energy is denoted by $K_{\vec{q}} \cdot E_{R}$ is the location of the resonance and $\Gamma$ is its width as functions of nuclear geometry. The energy, $E$, is the energy of the entire system, namely that of the target molecular state plus the kinetic energy of the incident electron. The driving term $\phi_{\nu_{i}}$ is proportional to the square root of the width times the initial vibrational wavefunction.

We have used our previously calculated [18] complexvalued potential energy surfaces for the three metastable electronic states. As in our previous calculations of the two-body cross sections [19], we used the diabatized ${ }^{2} \mathrm{~A}_{1}$ and ${ }^{2} \mathrm{~B}_{2}$ surfaces that account for the conical intersection between these states. The nuclear wave equation, Eq. (1), can be solved using time-dependent methods by representing the nuclear Green's function $(E-H+i \epsilon)^{-1}$ as the Fourier transform of the corresponding propagator [25]. The time-dependent nuclear dynamics were calculated using the Multi-Configuration Time-Dependent Hartree algorithm [26-28] and the numerical implementation by the group at Heidelberg, Germany [29].

In our previous calculations on two-body breakup, we employed Jacobi coordinate systems $(R, r, \gamma)$ based upon the diatomic fragment to be analyzed. Such a coordinate system is inappropriate for three-body breakup, as the asymptotic region is not well-defined in terms of a single degree of freedom. Instead, we use Delves-type [30, 31] hyperspherical coordinates, in which there is only one dissociative degree of freedom, the hyperradius $\mathbf{R}$. These coordinates are based upon the Jacobi coordinate system in which $r$ is an $\mathrm{OH}$ bond length and $R$ connects the $\mathrm{OH}$ center of mass with the other $\mathrm{H}$, and include the Jacobi angle $\gamma$, a hyperangle $\theta$, and the hyperradius $\mathbf{R}$ :

$$
\mathbf{R}=\sqrt{R^{2}+\frac{\mu_{r}}{\mu_{R}} r^{2}} \quad \theta=\tan ^{-1} \sqrt{\frac{\mu_{r}}{\mu_{R}}} \frac{R}{r},
$$

where the reduced masses correspond to the Jacobi coordinates. Further details of our numerical implementation will be forthcoming in a future publication[32].

In order to calculate a three-body breakup cross section, our strategy is to calculate, in hyperspherical coordinates, the total DEA cross section and then subtract the two-body cross sections from the total cross section. As in our earlier studies [15, 19], the two-body cross sections were obtained from the outgoing flux projected onto the bound rovibrational states of $\mathrm{OH}$ or $\mathrm{H}_{2}$. For the

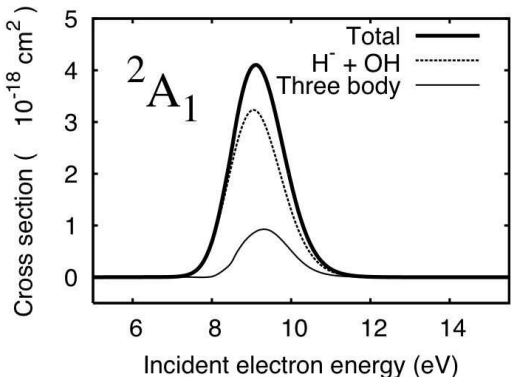

FIG. 1: Two- and three-body cross sections calculated for $\mathrm{DEA}$ via the ${ }^{2} \mathrm{~A}_{1}$ state.

present study, we have fully converged the $\mathrm{H}^{-}+\mathrm{OH} \mathrm{Ja}-$ cobi coordinate calculations for DEA via the ${ }^{2} \mathrm{~B}_{2}$ state and may now better resolve the branching ratio between $\mathrm{H}^{-}+\mathrm{OH}\left({ }^{2} \Sigma\right)$ and $\mathrm{H}^{-}+\mathrm{OH}\left(\mathrm{X}^{2} \Pi\right)$. The ${ }^{2} \mathrm{~A}_{1}$ calculation for this coordinate system was also redone. The $\mathrm{O}^{-}$ $+\mathrm{H}_{2}$ results are unchanged.

The three-body breakup threshold is $8.04 \mathrm{eV}$ for production of $\mathrm{H}+\mathrm{H}+\mathrm{O}^{-}$, and $8.75 \mathrm{eV}$ for $\mathrm{H}^{-}+\mathrm{H}+\mathrm{O}$; the onsets for the experimental peaks for $\mathrm{O}^{-}$and $\mathrm{H}^{-}$via ${ }^{2} A_{1}$ are approximately 7.9 and $7.5 \mathrm{eV}$, respectively[10]. Our potential energy surfaces, however, do not distinguish between the three-body asymptotes. As described in a previous publication[16], the full set of curves affecting the dynamics on the three resonant curves numbers at least five; there are eleven distinct seams of intersection among these surfaces. Our surfaces do not describe every one of these features. On the ${ }^{2} \mathrm{~A}_{1}\left(1^{2} \mathrm{~A}^{\prime}\right)$ surface, the $\mathrm{OH}+\mathrm{H}^{-}$and $\mathrm{H}+\mathrm{OH}^{-}$curves intersect as a function of $\mathrm{OH}$ distance, which will lead to coupling between these channels for high $\mathrm{OH} / \mathrm{OH}^{-}$vibrational levels. However, as the interacting curves are nearly parallel, we do not expect this feature of the physical surfaces to affect the branching ratio between three- and two-body breakup, except near onset. We have patched our calculated surface so that its asymptote lies exactly between the two physical asymptotes. Because our studies of the twobody channels in DEA via the ${ }^{2} \mathrm{~A}_{1}$ state yielded zero $\mathrm{O}^{-}$ $+\mathrm{H}_{2}$ cross section, we make the hypothesis that the $\mathrm{O}^{-}$ observed for DEA via the ${ }^{2} \mathrm{~A}_{1}$ resonance is entirely due to three-body dissociation.

While we expect the approximation to the ${ }^{2} \mathrm{~A}_{1}$ surface to have little influence on our results for three-body breakup, the true ${ }^{2} \mathrm{~B}_{2}$ surface has a feature that we have not included in the calculated surface, which may affect the dynamics significantly. As explained in Ref. [16], this feature is unique to metastable states, not being possible for bound states. It is a branch-point degeneracy seam with another metastable ${ }^{2} \mathrm{~B}_{2}$ state that leads to a double-valuedness of the surface; a transit about the seam exchanges the states. This seam is analagous to either member of the double-seams in Refs. [33, 34]. The two three-body asymptotes of this surface are $\mathrm{O}^{-}+\mathrm{H}$ $+\mathrm{H}$ and $\mathrm{O}\left({ }^{2} \mathrm{D}\right)+\mathrm{H}^{-}+\mathrm{H}$. The seam intersects the 


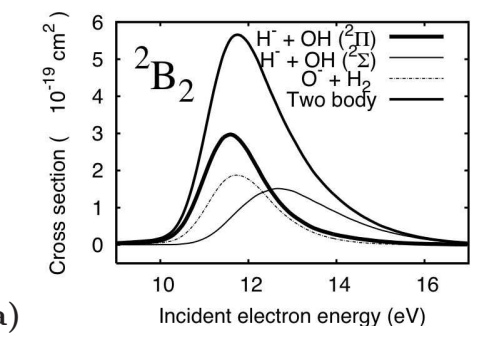



b)

c)

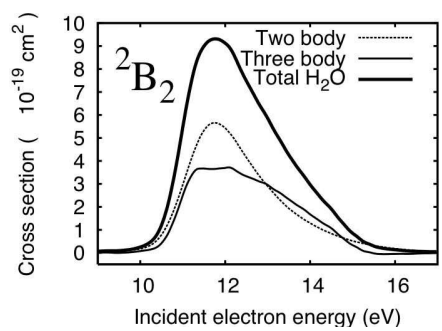

FIG. 2: Results for DEA via the ${ }^{2} \mathrm{~B}_{2}$ state, coupled to the ${ }^{2} \mathrm{~A}_{1}$ state via the conical intersection. a) Two-body cross sections. b) Kinetic energy release for DEA leading to $\mathrm{H}^{-}+\mathrm{OH}$. The cross section per unit kinetic energy release is plotted versus incident energy and kinetic energy of $\mathrm{H}^{-}+\mathrm{OH}$ separation. The cut at $12 \mathrm{eV}$ is compared with the experimental results of Belic, Landau, and Hall [8] in the inset. c) Two- and three-body cross sections.

$\mathrm{C}_{2 v}$ plane at a moderately stretched and squeezed geometry [16]. Since the gradient of the ${ }^{2} \mathrm{~B}_{2}$ surface will force the system towards this geometry, it is likely that the double-valuedness of the surface, and our approximation to it, will affect the dynamics. However, it is unclear how the approximation would affect three-body breakup. There is no experimental evidence for production of $\mathrm{O}\left({ }^{2} \mathrm{D}\right)$; however, the upper component of the double-valued set has a short lifetime and therefore it is likely that any $\mathrm{O}\left({ }^{2} \mathrm{D}\right)$ produced is not accompanied by an $\mathrm{H}^{-}$anion and is therefore invisible in the experiments performed to date. We truncate the ${ }^{2} \mathrm{~B}_{2}$ surface to its lower three-body asymptote, $\mathrm{O}^{-}+\mathrm{H}+\mathrm{H}$.

In Fig. 1 we plot the results for DEA via the ${ }^{2} \mathrm{~A}_{1}$ state. We calculate a significant three-body cross section. We assign our calculated ${ }^{2} \mathrm{~A}_{1}$ three-body breakup cross section to the $\mathrm{O}^{-}$channel. This is consistent with the topology of the surfaces, as direct symmetric dissociation of the ${ }^{2} \mathrm{~A}_{1}$ state leads to the $\mathrm{O}^{-}+\mathrm{H}+\mathrm{H}$ asymptote. The $\mathrm{O}$ $+\mathrm{H}^{-}+\mathrm{H}$ asymptote, on the other hand, is reached near the $\mathrm{OH}+\mathrm{H}^{-}$well, where the surface interacts with the $\mathrm{OH}^{-}+\mathrm{H}$ charge exchange state. The two-body cross section is entirely $\mathrm{H}^{-}+\mathrm{OH}$, our calculations yielding zero $\mathrm{O}^{-}+\mathrm{H}_{2}$ production.

In Fig. 2a we plot the new results for two-body breakup via the ${ }^{2} \mathrm{~B}_{2}$ resonance. These results show that, at onset, the cross section is dominated by $\mathrm{O}^{-}+\mathrm{H}_{2}$ and $\mathrm{H}^{-}+\mathrm{OH}$ $\left(\mathrm{X}^{2} \Pi\right)$, the lower-energy channels. The higher $\mathrm{H}^{-}+\mathrm{OH}$ $\left({ }^{2} \Sigma\right)$ channel dominates at high energy. Thus, the corresponding branching ratios are energy-dependent; the incident electron energy affects the probability of transit through the conical intersection.

We show the kinetic energy release for $\mathrm{H}^{-}+\mathrm{OH}\left({ }^{2} \Sigma\right.$, $\left.{ }^{2} \Pi\right)$ production calculated for DEA via the ${ }^{2} \mathrm{~B}_{2}$ state in Fig. 2b. This is a two-dimensional view of the $\mathrm{OH}$ peaks in Figure 2a. The solid lines indicate the maximum kinetic energy available, given that the diatomic fragment is in its ground rovibrational state. The upper area of contours shows the production of $\mathrm{H}^{-}+\mathrm{OH}\left(\mathrm{X}^{2} \Pi\right)$ from

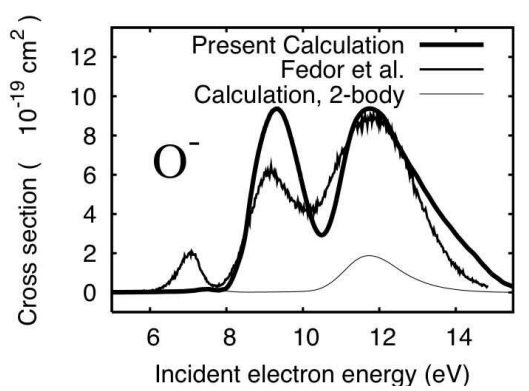

FIG. 3: Final results for $\mathrm{O}^{-}$production in DEA to the water molecule, with the experimental results of Fedor et al.[10].

dynamics leading through the conical intersection. The lower area corresponds to $\mathrm{H}^{-}+\mathrm{OH}\left({ }^{2} \Sigma\right)$ from dynamics avoiding the conical intersection. We calculate that the two-body cross sections for both $\mathrm{OH}\left(\mathrm{X}^{2} \Pi\right)$ and $\mathrm{OH}$ $\left({ }^{2} \Sigma\right)$ are dominated by diatoms in low-energy rovibronic states with high kinetic energy release. In this respect we confirm the results of Belic, Landau, and Hall [8], shown in the inset of Fig. 2b. We note that the $\mathrm{H}_{2}$ obtained for this resonance is produced in high rovibrational states, with low kinetic energy release [19].

These observations support a conclusion about the dynamics: the production of $\mathrm{O}^{-}+\mathrm{H}+\mathrm{H}$ via the ${ }^{2} \mathrm{~B}_{2}$ resonance is due to the vibrational continuum of $\mathrm{H}_{2}+$ $\mathrm{O}^{-}$and not that of $\mathrm{OH}+\mathrm{H}^{-}$(interacting with $\mathrm{OH}^{-}$ $+\mathrm{H})$. In other words, the dynamics does not follow the asymmetric path into one $\mathrm{OH}$ well and then outwards to three-body breakup; instead, $\mathrm{O}^{-}+\mathrm{H}+\mathrm{H}$ is produced along with $\mathrm{O}^{-}+\mathrm{H}_{2}$ in what is probably a more symmetric dissociation path.

In Figure 2c we show the two- and three-body cross sections for DEA via the ${ }^{2} \mathrm{~B}_{2}$ state. These results indicate that these channels strongly compete, as their cross sections have similar magnitude for all energies.

Our final results for $\mathrm{O}^{-}$production in DEA to the 
water molecule are summarized and compared with the recent results of Fedor et al.[10] in Fig.3. The ${ }^{2} \mathrm{~B}_{1}$ result is unchanged from our zero result $[19] ; \mathrm{O}^{-}$is the most minor channel $\left(\frac{1}{40}\right.$ branching ratio) in this system and represents the only qualitative failure of our treatment.

The resulting peak for $\mathrm{O}^{-}$production via the ${ }^{2} \mathrm{~A}_{1}$ state, at approximately $9.5 \mathrm{eV}$, is significantly larger than the experimental peak, just like our calculated $\mathrm{H}^{-}$peak for this resonance [19]. We may have overestimated the magnitude of the entrance amplitude (we have calculated the partial width to be $10.30 \mathrm{meV}$ [18]), or overestimated the survival probability. Both probabilities are directly affected by the lifetime of this metastable state as a function of nuclear geometry: a large entrance amplitude implies a small survival probability, and vice-versa. However, the ${ }^{2} \mathrm{~A}_{1}$ state becomes a virtual state at some geometries, and this fact has not been included in the current local complex potential model description. Virtual state effects may lead to an enhanced autodetachment probability and a lower survival probability, i.e., may account for our overestimation of the physical cross section.

The three-body calculations on the ${ }^{2} \mathrm{~B}_{2}$ state resolve a significant discrepancy between our prior results for $\mathrm{O}^{-}$production [19] and that obtained by experiment; the magnitude of this peak is now reproduced accurately. Disagreement in the high-energy tail region may be due to the fact that the present calculation does not account for decay of the the ${ }^{2} \mathrm{~B}_{2}$ state to the two-electron continuum.

Although it is not clear that our calculations within the local complex potential model include all the physical effects relevant to DEA of water, they appear to have reproduced the major features of experiment well, and demonstrate that three-body breakup is a major component of the observed $\mathrm{O}^{-}$cross section.

Most of this work was performed under the auspices of the US Dept. of Energy by the University of California Lawrence Berkeley National Laboratory under contract DE-AC02-05CH11231 and was supported by the U.S. DOE Office of Basic Energy Sciences. DJH acknowledges additional support at JILA, CU-Boulder, under DOE grant number W-31-109-ENG-38 and NSF grant number ITR 0427376. CWM acknowledges support from the National Science Foundation under grant number PHY0604628. DJH thanks JILA's Center for Atomic, Molecular, and Optical Physics, funded by the NSF's Physics Frontier Center Program, for computer resources.
[1] W. N. Lozier, Phys. Rev. 36, 1417 (1930).

[2] I. S. Buchel'nikova, Zh. Eksperim. i Teor. Fiz. 35, 1119 (1959).

[3] R. N. Compton and L. G. Christophorou, Phys. Rev. 154, 110 (1967).

[4] C. E. Melton, J. Chem. Phys. 57, 4218 (1972).

[5] L. Sanche and G. J. Schultz, J. Chem. Phys. 58, 479 (1972).

[6] S. Trajmar and R. I. Hall, J. Phys. B. 7, L458 (1974).

[7] M. Jungen, J. Vogt, and V. Staemmler, Chem. Phys. 37, 49 (1979).

[8] D. S. Belic̀, M. Landau, and R. I. Hall, J. Phys. B. 14, 175 (1981).

[9] M. G. Curtis and I. C. Walker, J. Chem. Soc. Faraday Trans. 88, 2805 (1992).

[10] J. Fedor, P. Cicman, B. Coupier, S. Feil, M. Winkler, K. Gluch, J. Husarik, D. Jaksch, B. Farizon, and N. J. Mason, J. Phys. B 39, 3935 (2006).

[11] C. R. Claydon, G. A. Segal, and H. S. Taylor, J. Chem. Phys 54, 3799 (1971).

[12] T. J. Gil, T. N. Rescigno, C. W. McCurdy, and B. H. Lengsfield III, Phys. Rev. A 49, 2642 (1994).

[13] J. D. Gorfinkel, L. A. Morgan, and J. Tennyson, J. Phys. B 35, 543 (2002).

[14] D. J. Haxton, Z. Zhang, C. W. McCurdy, and T. N. Rescigno, Phys. Rev. A 69, 062713 (2004).

[15] D. J. Haxton, Z. Zhang, H.-D. Meyer, T. N. Rescigno, and C. W. McCurdy, Phys. Rev. A 69, 062714 (2004).

[16] D. J. Haxton, T. N. Rescigno, and C. W. McCurdy, Phys. Rev. A 72, 022705 (2005).

[17] D. J. Haxton, C. W. McCurdy, and T. N. Rescigno, Phys. Rev. A 73, 062724 (2006).

[18] D. J. Haxton, C. W. McCurdy, and T. N. Rescigno, Phys. Rev. A 75, 012710 (2007).
[19] D. J. Haxton, T. N. Rescigno, and C. W. McCurdy, Phys. Rev. A 75, 012711 (2007).

[20] D. T. Birtwistle and A. Herzenberg, J. Phys. B 4, 53 (1971).

[21] L. Dube and A. Herzenberg, Phys. Rev. A 20, 194 (1979).

[22] J. N. Bardsley and J. M. Wadehra, J. Chem. Phys. 78, 7227 (1983).

[23] T. F. O’Malley, Phys. Rev. 150, 14 (1966).

[24] T. F. O'Malley and H. S. Taylor, Phys. Rev. 176, 207 (1968).

[25] C. W. McCurdy and J. L. Turner, J. Chem. Phys. 78, 6773 (1983).

[26] H.-D. Meyer, U. Manthe, and L. S. Cederbaum, Chem. Phys. Lett. 165, 73 (1990).

[27] U. Manthe, H.-D. Meyer, and L. S. Cederbaum, J. Chem. Phys. 97, 3199 (1992).

[28] M. H. Beck, A. Jackle, G. A. Worth, and H.-D. Meyer, Physics Reports 324, 1 (2000).

[29] G. A. Worth, M. H. Beck, A. Jäckle, and H.-D. Meyer, The MCTDH Package, Version 8.2, (2000). See http://www.pci.uni-heidelberg.de/tc/usr/mctdh/.

[30] L. M. Delves, Nucl. Phys. 9, 391 (1959).

[31] L. M. Delves, Nucl. Phys. 20, 275 (1960).

[32] D. J. Haxton, T. N. Rescigno, and C. W. McCurdy, in Multidimensional Quantum Dynamics: MCTDH Theory and Applications, edited by H.-D. Meyer, G. A. Worth, and F. Gatti (John Wiley \& Sons, Inc., Hoboken, NJ, to be published).

[33] S. Feuerbacher, T. Sommerfeld and L. S. Cederbaum, J. Chem. Phys. 120, 3201 (2004).

[34] S. Feuerbacher and L. S. Cederbaum, J. Chem. Phys. 121, 5 (2004). 